with the low temperature spectra shows that there are two lines remaining constant at different water contents, at 1534 and $1633 \mathrm{~cm}^{-1}$. In between one finds a broadband increasing in intensity and splitting into two lines at 1575 and 1591 $\mathrm{cm}^{-1}$ at higher water content.

(vi) At room temperature two broad lines arise at 3300 (assigned to $v \mathrm{NH}, \gamma \mathrm{NH}$, and $\tau \mathrm{CN}^{11}$ ) and $3103 \mathrm{~cm}^{-1}$ (assigned to $v \mathrm{NH}, \tau \mathrm{CN}^{11}$ ).

The question concerning the nature of these lines is not easy to answer. For such low concentrations of water (in NMA) one could first consider the vibrational lines of the isolated molecule, but not all possible lines (1595, 3151, and $3652 \mathrm{~cm}^{-1}$ ) ${ }^{16}$ fit to our observed ones. The problem seems to be more complicated as the water molecule will be bound in some way to the hydrogen bonds of the NMA crystal. One might even speculate that there are at least two different sites that are accessible to different numbers of water molecules. This could cause the different sensitivities of the two lines in the amide I/II region at room temperature. Further investigations on deuteration effects may lead to the necessary new assignments although the two factors of deuterating the amide group in NMA and water can only be switched at the same time because of the fast $H / D$ exchange between NMA and the added water.

We would like to thank A. Breitschwert for the operation of the spectrometer.
- Present address: Robert Bosch GmbH, Werk Schwieberdingen, Postfach 3002 40, 7000 Stuttgart 30, West Germany.

b) On leave from the Department of Chemistry, University of Oregon, Eugene, Oregon 97403.

'S. J. Mizushima, T. Shimanouchi, S. Nagakura, K. Kuratani, M. Tsuboi, H. Baba, and O. Fujioka, J. Am. Chem. Soc. 72, 3490 (1950).

${ }^{2}$ T. Miyazawa, T. Shimanouchi, and S. J. Mizushima, J. Chem. Phys. 24, 408 (1956); 29, 611 (1958).

${ }^{3}$ T. Miyazawa, J. Mol. Spectrosc. 4, 168 (1960).

${ }^{4}$ E. M. Bradbury and A. Elliott, Spectrochim. Acta 19, 995 (1963).

${ }^{5}$ B. Schneider, A. Horeni, H. Pivcova, and J. Honzl, Collect. Czech. Chem. Commun. 30, 2196 (1965).

${ }^{6} \mathrm{H}$. Pivcova, B. Schneider, and J. Stokr, Collect. Czech. Chem. Commun. 30, 2215 (1965).

${ }^{7} \mathrm{~J}$. Jakes and S. Krimm, Spectrochim. Acta 27, 19 (1971).

${ }^{8}$ G. Dellepiane, S. Abbate, P. Bosi, and G. Zerbi, J. Chem. Phys. 73, 1040 (1980).

${ }^{9}$ G. Dellepiane, P. Piaggio, M. Rui, and G. Zerbi, J. Mol. Struct. 61, 343 (1980).

${ }^{10}$ F. Fillaux and M. H. Baron, Chem. Phys. 62, 275 (1981).

"F. Fillaux, Chem. Phys. 62, 287 (1981).

${ }^{12}$ F. Fillaux, M. Jaber, and M. H. Baron, in Symmetries and Properties of Nonrigid Molecules: A Comprehensive Survey, edited by J. Maruani and J. Serre (Elsevier, Amsterdam, 1983), Vol. 23, pp. 247-256.

${ }^{13} \mathrm{G}$. Zerbi and J. Dellepiane, Raman Spectrosc. 12, 165 (1982).

${ }^{14} \mathrm{H}$. Hummel and R. Bonart, Makromol. Chem. 184, 2693 (1983).

${ }^{15}$ S. Häseler, A. Poglitsch, L. Genzel, and F. Kremer, Biopolymers (submitted).

${ }^{16} \mathrm{G}$. Herzberg, Molecular Spectra and Molecular Structure II. Infrared and Raman Spectra of Polyatomic Molecules (Van Nostrand Reinhold, New York, 1945).

\title{
Fractal behavior of correlated random walk on percolating clusters
}

\author{
Panos Argyrakis \\ Department of Physics, University of Crete, Iraklion, Crete, Greece \\ Raoul Kopelman \\ Department of Chemistry, University of Michigan, Ann Arbor, Michigan 48109
}

(Received 2 May 1985; accepted 2 October 1985)

Correlation in diffusional motion has been shown in the past to be a necessary idea for the explanation of experimental data ranging from the diffusion of hydrogen in metals ${ }^{1}$ and models of diffusion in concentrated lattice gases, ${ }^{2}$ to the relaxation mechanism of low-lying excited states of organic molecules at low temperatures ${ }^{3}$ as studied by the use of random walk hopping models. ${ }^{4}$ Recently a new model was introduced ${ }^{6,7}$ that incorporates the effects of correlation in the usual $^{5}$ random walk models, first on perfect lattices, ${ }^{6}$ and then on mixed binary lattices. ${ }^{7}$ Also of interest ${ }^{8}$ has been the application of the simple stochastic random walk on the fractal structures of percolating clusters around the critical threshold point. In the present paper we study the behavior of correlated random walks on such fractal structures, we test the possible universality of these phenomena, and also their crossover to the classical limit of a perfect crystal.
Percolating clusters are generated by a Monte-Carlo simulation method, using the cluster growth technique. ${ }^{9}$ The only prescribed parameter is the concentration (relative fraction) of the open (allowed) lattice sites $C$, ranging from the critical value $C_{c}=0.593$, to the limit of perfect lattice $C=1.00$. Correlation is the retention of the directional memory over a certain number of lattice spacings. This is quantitatively described by the fraction $p_{f}$, which is the probability of a forward jump, and it is in the range: $a^{-1}<p_{f}<1.00$, where $a$ is the lattice coordination number.

The well-known relation ${ }^{8}$ connecting $S_{N}$, the number of distinct sites visited in an $N$-step walk, with $N$ (time) is

$$
S_{N} \sim N^{d_{s} / 2}
$$

which has been shown' to hold true for a variety of lattices for stochastic random walks with a $d_{s}$ value: $d_{s}^{e}=1.30$, for 
a random walk restricted to the largest cluster only, while $d_{s}^{\prime}=1.23$, for the random walk originating on any cluster, small or large, ${ }^{10}$ all for the square two-dimensional lattice. This is exhibited by the curve marked $p_{f}=0.25$ in Fig. 1. In this case of the square lattice all four directions carry the same 0.25 probability of scattering, so that this is the limit of a totally uncorrelated walk. All calculations shown in Fig. 1 are performed using random walks that may start on any size clusters, so that exactly at the critical point $\left(C_{c}=0.593\right)$ the (modified ${ }^{10,11}$ ) spectral dimension $d_{s}^{\prime}=1.23$. However, for $C=0.60$, we observe that $d_{s}^{\prime}=1.33$, showing that we are already in the region of "crossover" to Euclidean behavior. As has been recently shown, ${ }^{9}$ this crossover is very sudden close to the critical point, so that the observed difference (1.23 to 1.33 ) is not surprising, but rather in agreement with these recent results. We observe that the standard deviations for all the above values are about \pm 0.02 . We further observe that the crossover to Euclidean behavior eventually $(C=1.00)$ results in the value of $d_{s}^{e}=1.8$. (Here $d_{s}^{e}$ is an effective spectral dimension; as is well known, the difference from the $d_{s}=2$ classical value is due to the logarithmic correction terms that the two-dimensional random walk exhibits. $^{5}$ )

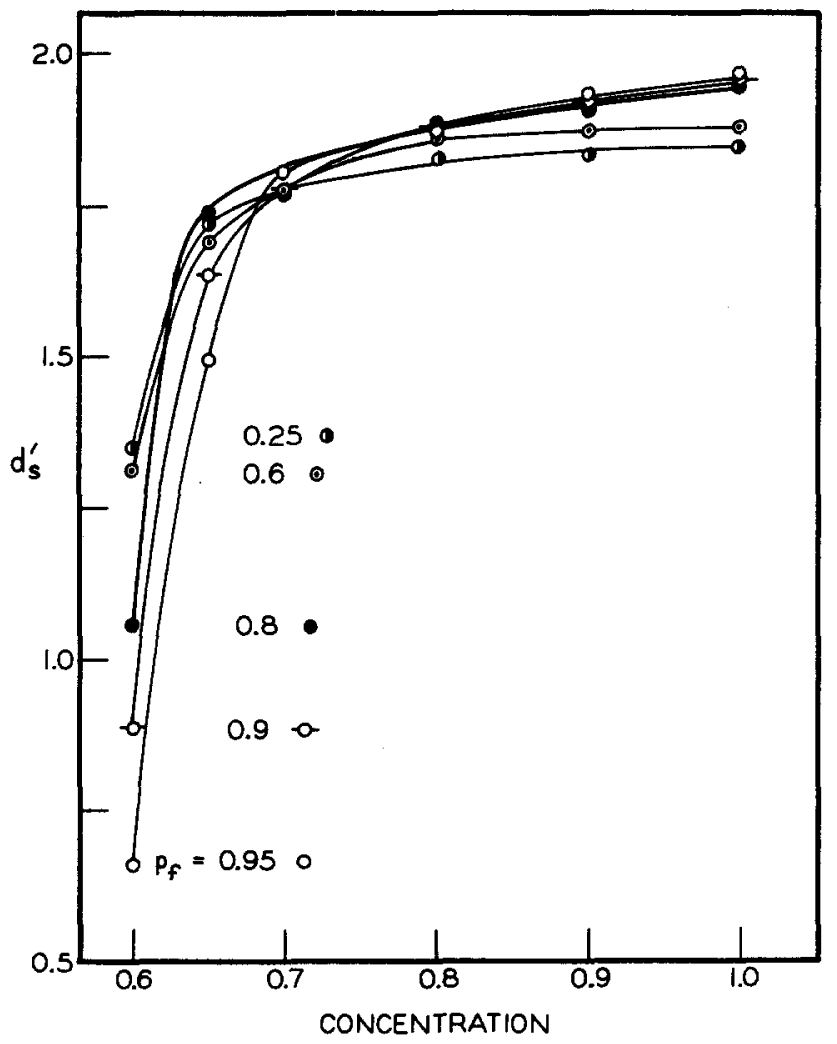

FIG. 1. The spectral dimension $d_{s}^{\prime}$ vs the concentration $C$, as a function of the forward correlation parameter $p_{f}$. These are results of random walks on lattices of $2000 \times 2000$, averaged over 500 realizations, and the spectral dimensions are calculated in the long time limit, $N=2 \times 10^{5}$ steps, from walks that may originate on any size cluster.
Of special interest here are the other curves in Fig. 1, which refer to higher $p_{f}\left(p_{f}>0.25\right)$ values. In the fractal limit $(C=0.60)$ we see that $d_{s}^{\prime}$ sharply decreases as $p_{f}$ increases. For $p_{f}=0.95$ (at $C=0.60$ ) we see that $d_{s}^{\prime}=0.66$ only. This sharp decrease accompanies the fact that at the critical percolation threshold the correlated walks have a much smaller $S_{N}$ value than the uncorrelated walks, because, as was originally shown in the past, ${ }^{4}$ the particle indulges for long times in revisiting the same row of sites over and over again. We also used this idea ${ }^{3}$ to interpret experimental data on mixed naphthalene alloys at $2 \mathrm{~K}$. The trend in this decrease is not simple, however. Actually for small $p_{f}$, in the region $0.25<p_{f}<0.50$, we observe an increase in the $d_{s}^{\prime}$ value compared to $p_{f}=0.25$, coming from a corresponding increase in the $S_{N}$ respective values. Only for high $p_{f}$, i.e., $p_{f}>0.60$, do we observe a clear decrease in the spectral dimension, resulting from a drastic decrease in $S_{N}$. This shows that the problem is considerably more complex than Fig. 1 shows, but its details are not to be addressed here. Thus, our preliminary conclusion is that correlated walks do not belong to the universality class of stochastic walks. Factors that have to be taken into account include: the exact value of $C$ (remembering that $C=0.60$ is not the critical point but $C_{c}=0.593$ ), the length of time, contributions from runs on small finite clusters, the use of the "blind" or "myopic" ant model, ${ }^{12}$ etc.

As $C$ increases, one observes for each $p_{f}$ value the corresponding crossover to the classical behavior, since now, above $C=0.80$, correlated walks are much more efficient than uncorrelated ones. The region $0.70<C<0.80$, as seen from Fig. 1, is the "crossover region" between the different $p_{f}$ values.

In summary, we have presented a preliminary study for correlated random walk motion on fractal structures, and we investigated the two types of crossover that occur, i.e., the crossover from fractal to Euclidean behavior for any type of walk, and the crossover from uncorrelated to correlated walk at any given concentration.

This work was supported by NATO Grant SA-5205RG-295/82 and NSF Grant DMR 8303919.

'J. W. Haus and K. W. Kehr, Solid State Commun. 26, 753 (1978); V. Lottner, J. W. Haus, A. Heim, and K. W. Kehr, J. Phys. Chem. Solids 40, 557 (1979); W. Gissler and H. Rother, Physica 50, 380 (1970).

${ }^{2}$ K. W. Kehr, J. Stat. Phys. 30, 509 (1983).

${ }^{3}$ P. Argyrakis and R. Kopelman, Chem. Phys. 57, 29 (1981); 78, 251 (1983).

${ }^{4}$ P. Argyrakis and R. Kopelman, J. Theor. Biol. 73, 205 (1978).

${ }^{5}$ A. Blumen and G. Zumofen, J. Chem. Phys. 75, 892 (1982); 76, 3713 (1982).

${ }^{6} \mathrm{~K}$. W. Kehr and P. Argyrakis, J. Chem. Phys. (submitted).

${ }^{7}$ P. Argyrakis and K. W. Kehr (to be published).

'S. Alexander and R. Orbach, J. Phys. Lett. 43, L625 (1982); R. Rammal and G. Toulouse, ibid. 44, L13 (1983).

${ }^{9}$ P. Argyrakis and R. Kopelman, J. Chem. Phys. 81, 1015 (1984); 83, 3099 (1985).

${ }^{10}$ This distinction between the two exponents that depends on the cluster of origin was first made by: D. Ben-Avraham and S. Havlin, J. Phys. A 15, L691 (1982); 15, L311 (1982).

${ }^{11}$ I. Webman, Phys. Rev. Lett. 52, 220 (1984).

${ }^{12}$ C. D. Mitescu and J. Roussenq, Ann. Isr. Phys. Soc. 5, 81 (1983). 